\title{
Some Features of the Investment Process and International Investment Projects in the Conditions of Bulgarian Economy and Politics
}

\author{
Ivo Veselinov Yotsov and Siyana Lutzkanova* \\ Nikola Vaptsarov Naval Academy \\ 73 Vasil Drumev Str., Varna, 9026 Bulgaria
}

Received 20.01.2015, received in revised form 06.10.2015, accepted 10.01.2016

The focus is on the analysis of data on FDI in Bulgaria during the period 1998-2013, and the issues related to characteristics of the host country and relative advantages of the allocation of the business activity of foreign investors in Bulgaria The historical transition that Bulgaria made from a planned to a market economy provides a rich research material of the impact of the risk on the national development of the investment process and in particular, the dynamics of foreign direct investment. Trends were examined about the foreign direct investment during the period 1999-2013, based on the official data from the Bulgarian National Bank (BNB), UNCTAD and the Bulgarian Investment Agency. The analysis revealed three main periods, first from 1999 to 2003, from 2004 to 2007 and the third - from 2008 to 2013, characterized by a sharp decrease in the flow of foreign investments. The low level of FDI in the period before accession to the EU and NATO is a result of the high national risk in all its aspects - political, economic, financial and cultural. The significant growth of FDI in the period 2005-08 was due to the overall condition of the economic cycle, the internal and external political, economic and financial stability.

Keywords: Foreign Direct Investment, Economic Growth, Regional Economic Activity.

DOI: 10.17516/1997-1370-2016-9-2-325-341.

Research area: economics, politology.

Economic and public interest during the period of market reforms in $90^{\text {'s }}$ of the 20th century imposed meant further research in the field of Foreign Direct Investment (FDI) in the countries with economies in transition, including Bulgaria. Significant inflow of FDI in the Bulgarian economy was observed in the period 1998-2006, prior to the accession of Bulgaria to the European Union. The peak of FDI in Bulgaria was in $2007^{1}$.
Analysis of data about FDI in Bulgaria during the period 1998-2013 is concentrated on the characteristics of the business environment in Bulgaria and its comparative advantages, determining the flow and preferences (regional and sectoral) of the foreign investors.

The characteristics by which United Nations Conference on Trade and Development (UNCTAD) estimates countries in the field of attracting FDI are the index of investment

(C) Siberian Federal University. All rights reserved

* Corresponding author E-mail address: ivo.yotsov@nvna.eu, s.lutzkanova@abv.bg 
potential and index of FDI. The index of investment potential is based on several quantifiable indicators:

- GDP per capita,

- The rate of GDP growth over the last 10 years,

- The share of exports in GDP,

- Average number of telephone lines per 1,000 inhabitants

- Commercial energy use per capita,

- The share of $R \& D$ spending in GDP,

- The share of university graduates from the population,

- National risk,

- The market share in the global export of natural resources,

- The market share in the global import of parts and components for automobiles and electronic products

- The market share in the global export of services,

- The share of global FDI inward stock ${ }^{2}$

This index characterizes the actual investment opportunities offered by the individual countries and represents a quantitative and, to acertain degree, qualitative characteristics that takes into account the objective conditions for investment, based on the level of economic development, 3 as a basis for ranking. Index of FDI performance characterizes actual FDI inflow in a country and is determined by the formula:

$\operatorname{Ind}_{i}=\frac{F D I i / F D I w}{G D P i / G D P w}$

Where: FDI i-FDIin the country

FDIw-aggregateFDIin the world

GDP i-grossdomestic product

GDP w-aggregate gross domesticproduct in the world

The formula shows FDI ratio in the given country according to its place in the global economy. The comparison between these two indices enables the analysis of the effectiveness of the government policy in terms of attracting FDI. The quantitative indices used for the assessment of the index of investment potential are characterized with considerable stability and predictability. Most dynamic is the assessment of national risk as long as it depends on many factors that can be influenced by national governments. That is the main factor that often causes a significant difference between the two indices (see. Fig. 1) Therefore, we will examine how the national risk affects FDI inflow in Bulgaria during the period 1996-2013.

\section{Influence of the political risks on the dynamics of FDI in Bulgaria in the period 1996-2013}

According to BNB data, the net transactions of FDI in Bulgaria from 1996 to 2012 are worth 137.3 million Euro per year, while in 2007 they are 9051.8 million Euro, an increase of nearly 66 times. Sharp increase in FDI is observed within 2002-07 (Fig. 2) The low level of FDI in the period before 2002 is determined by a number of factors, such as political instability of the region, difficult to predict economic perspectives, lack or inefficiency of the structural reforms leading to malfunction of certain markets (or even, lack of markets), and last but not least, lack of professionals adequately trained to carry out the investment process. Despite the generally low level of FDI in certain periods, there are considerable monthly fluctuations in their dynamics (Fig. 3). That dynamics is closely linked to the political events both in the country and in the region (South-Eastern Europe and especially, the Balkan Peninsula).

Figure 4, showing the dynamics of FDI in Bulgaria in the period 1998-99, outlines the impact of the ultimate escalation of the conflict in the former Yugoslavia, when in the beginning of 1998 the Serbian army was deployed in Kosovo in 
Table 1. Top 20 rankings by FDI Performance Index ${ }^{1}$

\begin{tabular}{|c|c|c|c|}
\hline Economy & 1995 & $2002-2004$ & $2006-2008$ \\
\hline Hong Kong, China & 11 & 7 & 1 \\
\hline Bulgaria & 93 & 8 & 2 \\
\hline Congo & 8 & 41 & 3 \\
\hline Angola & 23 & 2 & 4 \\
\hline Belgium & & 10 & 5 \\
\hline Singapore & 2 & 6 & 6 \\
\hline Malta & 20 & 25 & 7 \\
\hline Guinea & 126 & 93 & 8 \\
\hline Georgia & 114 & 17 & 9 \\
\hline Jordan & 132 & 38 & 10 \\
\hline Guyana & 1 & 39 & 11 \\
\hline Panama & 31 & 37 & 12 \\
\hline Lebanon & 116 & 9 & 13 \\
\hline Madagascar & 110 & 95 & 14 \\
\hline Bahrain & 45 & 30 & 15 \\
\hline Gambia & 29 & 11 & 16 \\
\hline Estonia & 15 & 20 & 17 \\
\hline Moldova, Republic of & 60 & 44 & 18 \\
\hline Bahamas & 56 & 15 & 19 \\
\hline Kazakhstan & 19 & 13 & 20 \\
\hline
\end{tabular}

Sulstarova A., Division on Investment and Enterprise, UNCTAD

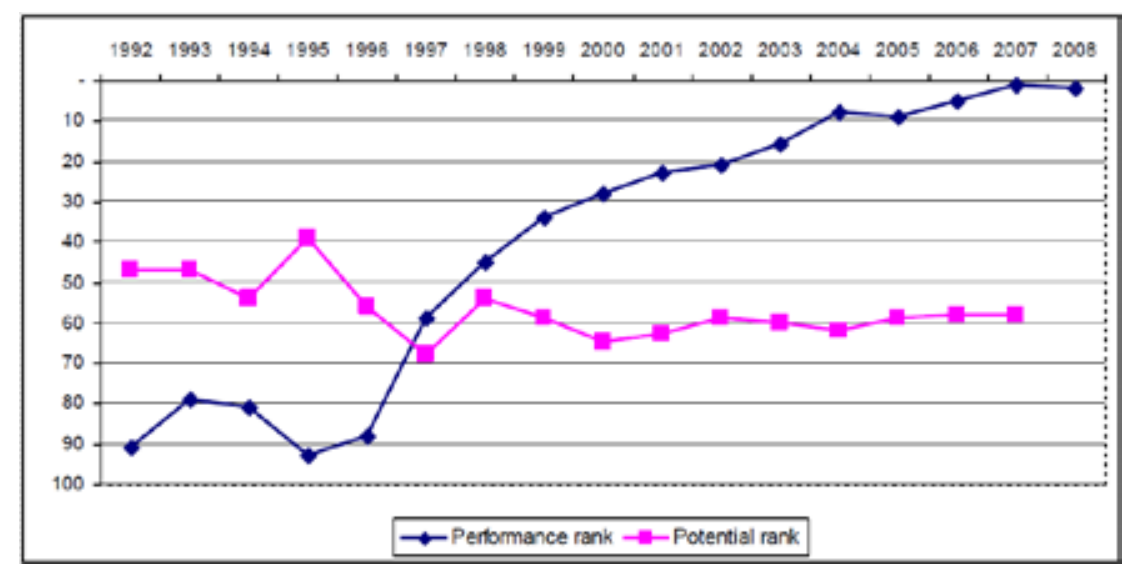

Fig. 1. Bulgaria - FDI Performance Index and FDI potential index ( Sulstarova A., Division on Investment and Enterprise, UNCTAD) 
Table 2. FDI Bulgaria 1998-2012, annual data, source Bulgarian National Bank

\begin{tabular}{|c|c|c|c|c|c|c|c|c|c|c|c|c|c|c|c|c|c|}
\hline $\begin{array}{l}\bar{O} \\
\stackrel{0}{0} \\
0\end{array}$ & ஃ̊ & $\hat{\circ}$ & $\stackrel{\infty}{\circ}$ & ڤ̆ & ঃ̊ & 홍 & ণิ & ֻ̊̊̊̊ & ঠి & 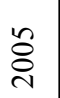 & ঃ̊ & 용 & $\stackrel{\infty}{\circ}$ & ஓి & $\stackrel{\circ}{\circ}$ & $\overline{\bar{\delta}}$ & 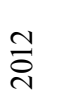 \\
\hline 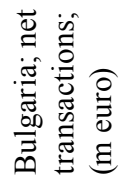 & $\stackrel{n}{n}$ & $\frac{N}{i n}$ & $\overrightarrow{0}$ & $\begin{array}{l}0 \\
0 \\
\infty \\
\infty\end{array}$ & $\begin{array}{l}2 \\
\hat{\varrho} \\
=\end{array}$ & $\stackrel{+}{\dot{\sigma}_{0}}$ & $\begin{array}{l}0 \\
\stackrel{0}{ } \\
\stackrel{0}{0}\end{array}$ & $\begin{array}{l}n \\
0 \\
\infty \\
\infty \\
\infty\end{array}$ & $\begin{array}{l}\hat{n} \\
\hat{\sim}\end{array}$ & $\vec{n}$ & 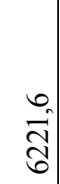 & $\begin{array}{l}\infty \\
\vec{n} \\
\stackrel{-}{2}\end{array}$ & $\stackrel{\infty}{\stackrel{\infty}{\widehat{\sigma}}}$ & $\begin{array}{l}\hat{\sigma} \\
\hat{0} \\
\stackrel{n}{+}\end{array}$ & $\begin{array}{l}\mathfrak{n} \\
\stackrel{n}{=}\end{array}$ & 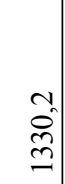 & $\begin{array}{l}n \\
2 \\
\infty \\
\pm \\
\pm\end{array}$ \\
\hline
\end{tabular}

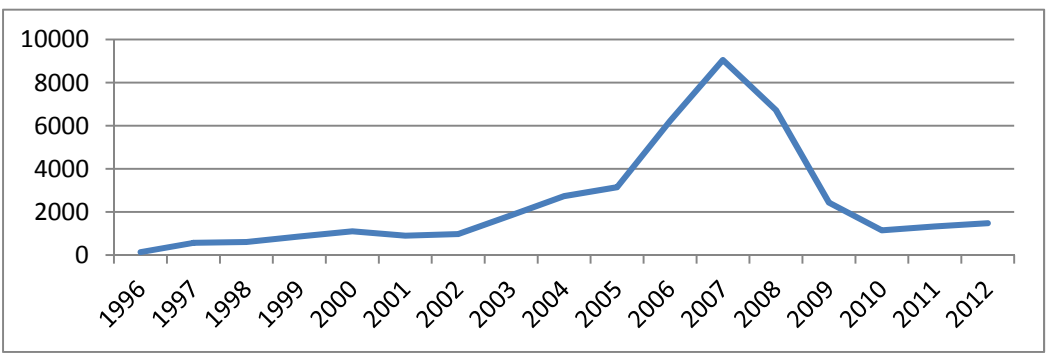

Fig. 2. FDI in Bulgaria, 1996-2012, annual data, source Bulgarian National Bank

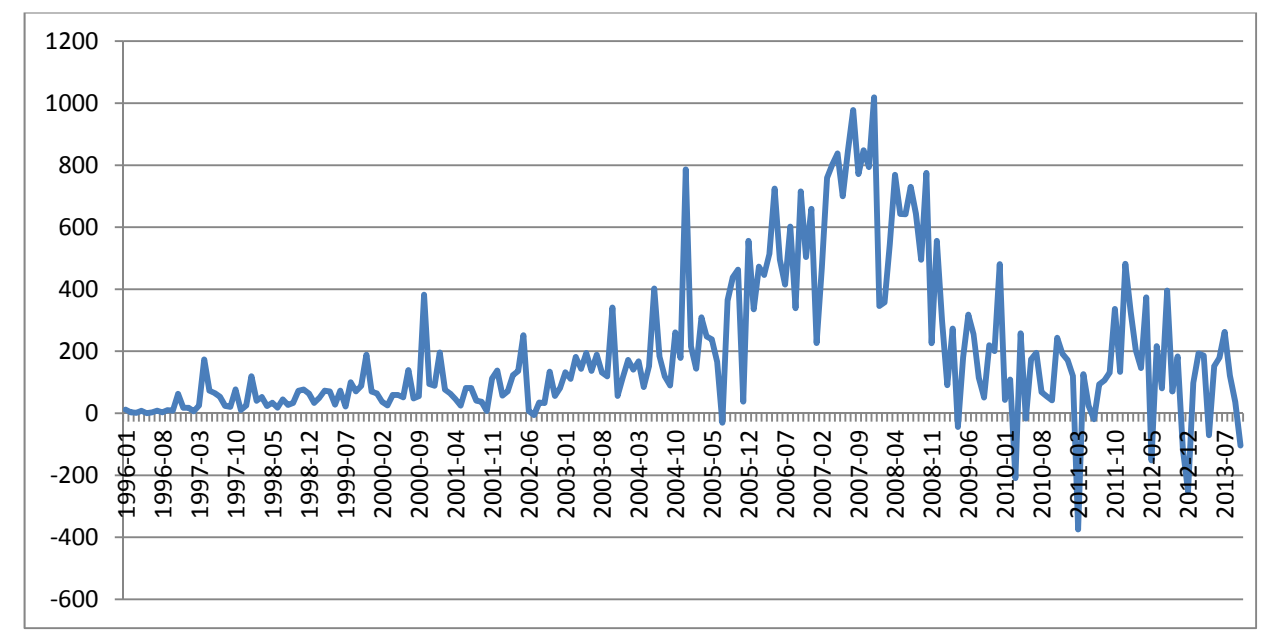

Fig. 3. FDI in Bulgaria, 1996-2012, monthly data, source Bulgarian National Bank

offensive against The Kosovo Liberation Army.

${ }^{4}$ Being a state neighbouring the conflict, these events affect the national risk of Bulgaria. The Bulgarian governmental policy to support NATO and especially, the declarations of the National Assembly on October 23, 1998 and March 25, 1999, had a positive impact on FDI. However, the national risk remains high, which is reflected in the dynamics of FDI.

Figure 5 demonstrates the dependency between the dynamics of net transactions of FDI in Bulgaria and the conflict in Macedonia (from the end of 2000 to January 2002). The conflict escalated in early 2001, when the 


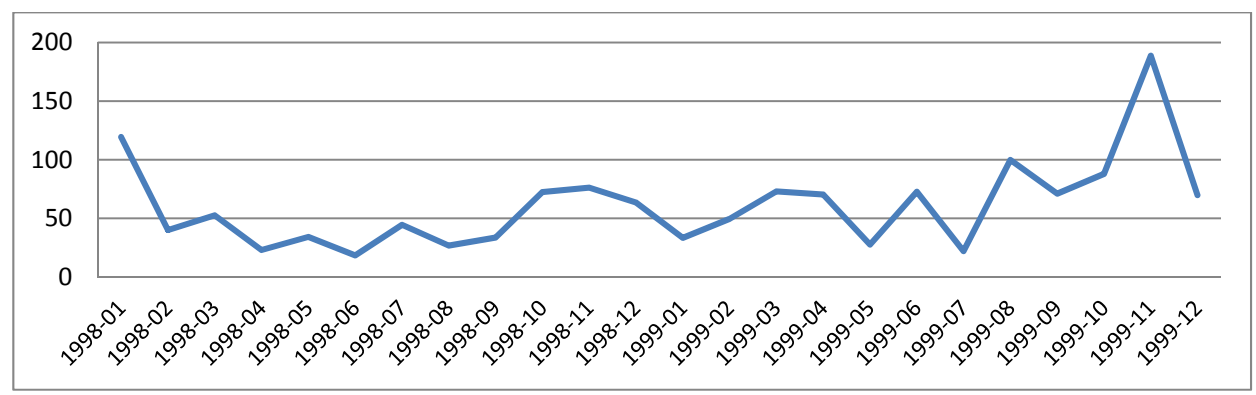

Fig. 4. Dynamics of FDI in Bulgaria within January1998 - December 1999 (m Euro), source Bulgarian National Bank

Table 3. FDI Bulgaria, 2000-2001, annual data, source Bulgarian National Bank

\begin{tabular}{|c|c|c|c|c|c|c|c|c|c|c|c|c|c|c|c|c|c|c|c|c|c|c|c|c|c|}
\hline \multirow[b]{2}{*}{$\begin{array}{l}\overrightarrow{0} \\
\stackrel{0}{0} \\
0\end{array}$} & \multicolumn{12}{|c|}{ ஓ } & \multicolumn{12}{|c|}{ 훙 } & ํ. \\
\hline & $\overline{0}$ & $\widetilde{O}$ & $\hat{0}$ & $\Xi$ & $\stackrel{2}{0}$ & ๖ & $\hat{o}$ & $\infty$ & 8 & 으 & $=$ & $\simeq$ & $\overline{0}$ & ป & $\hat{\sigma}$ & I & $\stackrel{2}{0}$ & 8 & $\hat{o}$ & $\stackrel{\infty}{\circ}$ & gे & 으 & $=$ & $\simeq$ & $\bar{o}$ \\
\hline 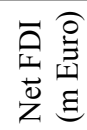 & $\begin{array}{l}m \\
\tilde{f}\end{array}$ & $\begin{array}{l}0 \\
\text { n. }\end{array}$ & $\stackrel{\nabla_{n}}{\sim}$ & $\begin{array}{l}\infty \\
\infty \\
\infty\end{array}$ & $\overrightarrow{a n}$ & $\frac{0}{n}$ & ๙ે & $\hat{\tilde{F}}$ & $\vec{n}$ & $\begin{array}{c}n \\
\tilde{\infty} \\
m\end{array}$ & $\stackrel{n}{\text { ga }}$ & $\begin{array}{l}n \\
\infty \\
\infty\end{array}$ & $\stackrel{\infty}{2}$ & $\begin{array}{l}\infty \\
n\end{array}$ & $\begin{array}{l}\infty \\
\hat{\sigma}\end{array}$ & $\begin{array}{l}\infty \\
\ddots \\
f\end{array}$ & $\stackrel{\vec{v}}{\sim}$ & $\frac{\sqrt{\infty}}{\infty}$ & $\underset{\infty}{+\infty}$ & ? & $\vec{n}$ & $\stackrel{m}{n}$ & $\stackrel{ }{\simeq}$ & $\stackrel{n}{n}$ & बें \\
\hline
\end{tabular}

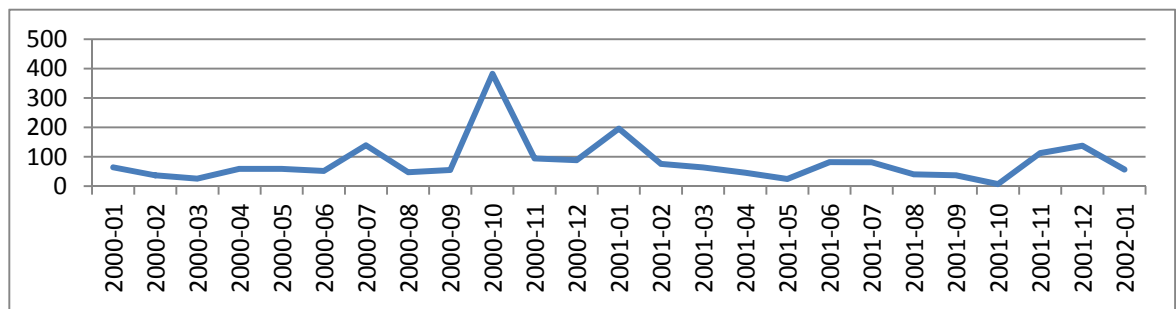

Fig. 5. FDI Bulgaria, 2000-01, monthly data, source Bulgarian National Bank

Albanian separatists, applying tactics used in Kosovo, started to take over village after village in North-Western Macedonia. Moreover, there was a high probability the conflict to develop in a manner similar to that in Kosovo. This is the reason for the sharp drop of FDI in Bulgaria in January 2001 and maintaining the descending trend until October 2001. In addition to the political volatility of the region, the period is characterized by a domestic politic crisis. On April 19, the $38^{\text {th }}$ National Assembly was suspended and parliamentary elections were scheduled (June 17, 2001). From July 2001 , the $39^{\text {th }}$ National Assembly began work with the government of Simeon Saxe-CoburgGotha. The slight increase in FDI in June and July can be explained with the likelihood for positive changes in the policy of the Bulgarian government, resulting from the recruitment of many young people to management who were 
not tied by the political and economic leadership of the country up to that point.

Since 2002, there is a steady trend of increased FDI in Bulgaria (see. Fig. 2). The period was characterized by political stabilization of the region and strengthening the process of EuroAtlantic integration of transition economies and of Bulgaria, in particular. The beginning of this process, for Bulgaria, started on May 8, 1997 with the adoption by the National Assembly of a Declaration of national consensus that defines the accession of Bulgaria to NATO as a major national priority. In October 2001, Sofia hosted the meeting of the presidents of the NATO countries. ${ }^{5}$ On March 18, 2004 the National Assembly of the Republic of Bulgaria ratified the North Atlantic Treaty, and on March 29, 2004 documents of accession to NATO of the seven new countries (Bulgaria, Estonia, Latvia, Lithuania, Slovakia, Slovenia and Romania) have been deposited in Washington. The final date is the date of accession of Bulgaria to NATO. This period is characterized by increasing integration of the Republic of Bulgaria and the EU, which ended in 25 April 2005 with the signing of the accession treaty to the European Union from January 1,2007 . The stabilization of ethnic rivalry in the region, integration in the Euro-Atlantic structures and structural reforms reflected in the continuous growth of FDI. From 980.0 m Euro value of FDI in 2002, we see almost $200 \%$ annual growth until 2007. The sharp drop of FDI after 2008 is the result of the action of a global factorThe global financial crisis of 2008.

The data on Figures 4 to 8 incl. shows an explicable regularity; since the dissolution of the National Assembly until the election of a new one and the appointment of government, there was a drop in FDI inflows. ${ }^{6}$ Interesting is the 40th National Assembly elected on June 25, 2005. Figure 6 is shows a sharp decline in FDI inflows resulting from the parliamentary crisis associated with the difficult choice of government caused by parity of forces in the parliament. 2013 is also a

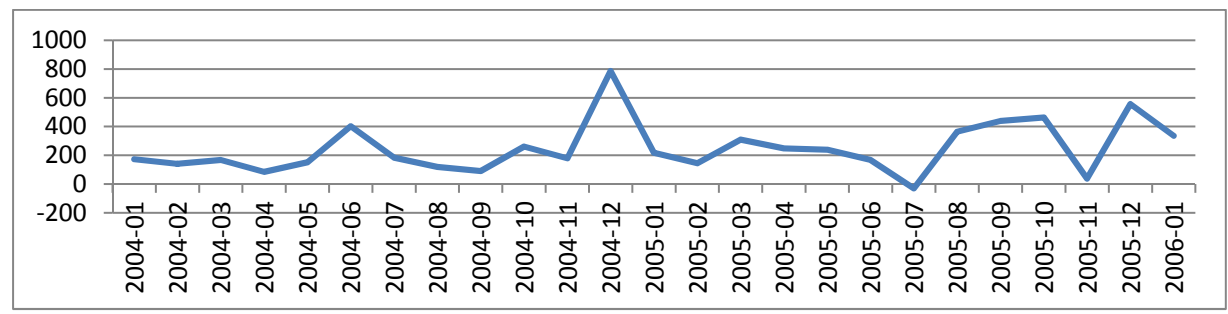

Fig. 6. FDI Bulgaria, 2004-05, monthly data, source Bulgarian National Bank

Table 4. FDI Bulgaria, 2006-2007, monthly data, source Bulgarian National Bank

\begin{tabular}{|c|c|c|c|c|c|c|c|c|c|c|c|c|c|c|c|c|c|c|c|c|c|c|c|c|}
\hline \multirow[b]{2}{*}{$\begin{array}{l}\overrightarrow{0} \\
.00 \\
0 \\
0\end{array}$} & \multicolumn{12}{|c|}{ ஓ̊ } & \multicolumn{12}{|c|}{ 옹 } \\
\hline & $\bar{\sigma}$ & ชิ & $\hat{0}$ & む & $\stackrel{n}{0}$ & 8 & $\hat{0}$ & $\stackrel{\infty}{\circ}$ & aे & & $=$ & $\simeq$ & $\overline{0}$ & $\widetilde{\delta}$ & $\hat{0}$ & む & 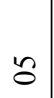 & 8 & $\hat{0}$ & 0 & वे & 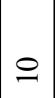 & $=$ & $\simeq$ \\
\hline 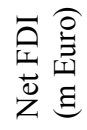 & ñ & $\begin{array}{l}0 \\
\stackrel{N}{f}\end{array}$ & $\overrightarrow{6}$ & $\frac{0}{i n}$ & $\stackrel{\mathbb{N}}{\mathbb{N}}$ & $\begin{array}{l}\dot{\sigma}^{2} \\
\stackrel{+}{\sigma}\end{array}$ & $\bar{\sigma}$ & $\frac{\sigma}{0}$ & $\begin{array}{c}\hat{\infty} \\
\infty \\
\tilde{m}\end{array}$ & $\vec{n}$ & $\begin{array}{l}0 \\
\text { ஸ் } \\
i n\end{array}$ & $\begin{array}{c}a \\
\infty \\
\sigma \\
\sigma\end{array}$ & 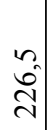 & $\begin{array}{l}\infty \\
\infty \\
\infty \\
\dot{y}\end{array}$ & $\stackrel{2}{2}$ & $\overrightarrow{\tilde{\delta}}$ & $\begin{array}{c}\hat{\sigma} \\
\hat{\infty}\end{array}$ & $\hat{\sigma}$ & $\begin{array}{c}n \\
\tilde{f} \\
\infty\end{array}$ & $\begin{array}{c}0 \\
\infty \\
5 \\
a\end{array}$ & $\frac{n}{2}$ & $\begin{array}{l}\sim \\
\infty \\
\infty \\
\infty\end{array}$ & 胥 & $\begin{array}{l}\tau_{0} \\
\stackrel{\infty}{0}\end{array}$ \\
\hline
\end{tabular}




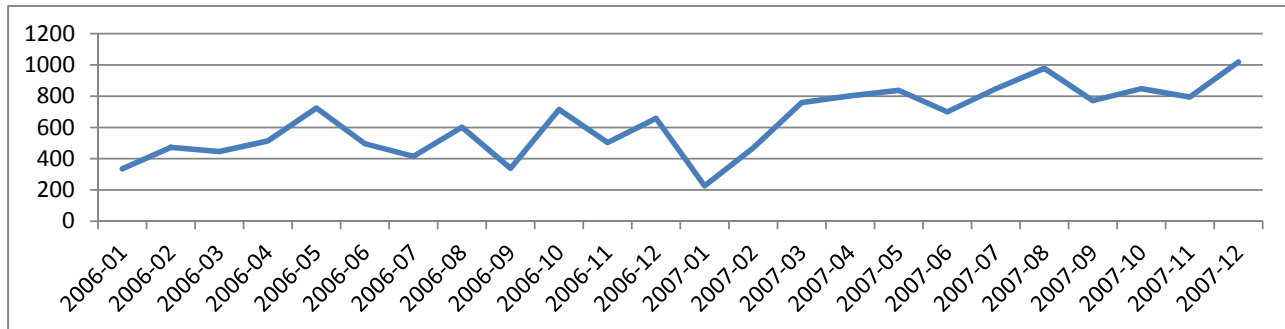

Fig. 7. FDI Bulgaria, 2006-2007, monthly data, source Bulgarian National Bank

Table 5. FDI Bulgaria, 2010-2011, monthly data, source Bulgarian National Bank

\begin{tabular}{|c|c|c|c|c|c|c|c|c|c|c|c|c|c|c|c|c|c|c|c|c|c|c|c|c|}
\hline \multirow[b]{2}{*}{$\begin{array}{l}\frac{D}{0} \\
\stackrel{0}{0} \\
0\end{array}$} & \multicolumn{12}{|c|}{$\stackrel{\circ}{\circ}$} & \multicolumn{12}{|c|}{$\overline{\bar{\sigma}}$} \\
\hline & $\bar{\sigma}$ & ชิ & \% & $\Xi$ & $\stackrel{n}{\circ}$ & \& & $\hat{0}$ & $\stackrel{\infty}{\circ}$ & gे & 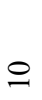 & $=$ & $\simeq$ & $\bar{\sigma}$ & ปู & $\tilde{\sigma}$ & $\Xi$ & 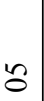 & 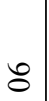 & $\hat{0}$ & $\stackrel{\infty}{\circ}$ & gे & 으 & $=$ & $\simeq$ \\
\hline 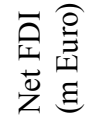 & $\vec{\vartheta}$ & $\hat{\infty}$ & $\overrightarrow{\sigma_{0}}$ & $\frac{n}{n}$ & $\frac{M}{1}$ & $\underline{\Xi}$ & $\begin{array}{l}\stackrel{0}{a} \\
\stackrel{2}{a}\end{array}$ & $\begin{array}{l}\text { Na } \\
\infty \\
0\end{array}$ & $\begin{array}{l}0 \\
\text { tr }\end{array}$ & $\bar{F}$ & 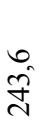 & $\stackrel{0}{a}$ & $\stackrel{n}{\approx}$ & 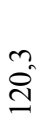 & $\begin{array}{l}0 \\
\frac{1}{1}\end{array}$ & $\stackrel{\overbrace{}}{\approx}$ & $\begin{array}{l}\stackrel{\sim}{\sim} \\
\stackrel{\sim}{*}\end{array}$ & $\begin{array}{l}0 \\
\frac{0}{1}\end{array}$ & $\begin{array}{l}0 \\
\text { ñ }\end{array}$ & $\stackrel{\infty}{\infty}$ & $\vec{\approx}$ & $\begin{array}{l}\text { ñ } \\
\text { mn } \\
\tilde{n}\end{array}$ & $\ddot{m}$ & $\overrightarrow{\tilde{\infty}}$ \\
\hline
\end{tabular}

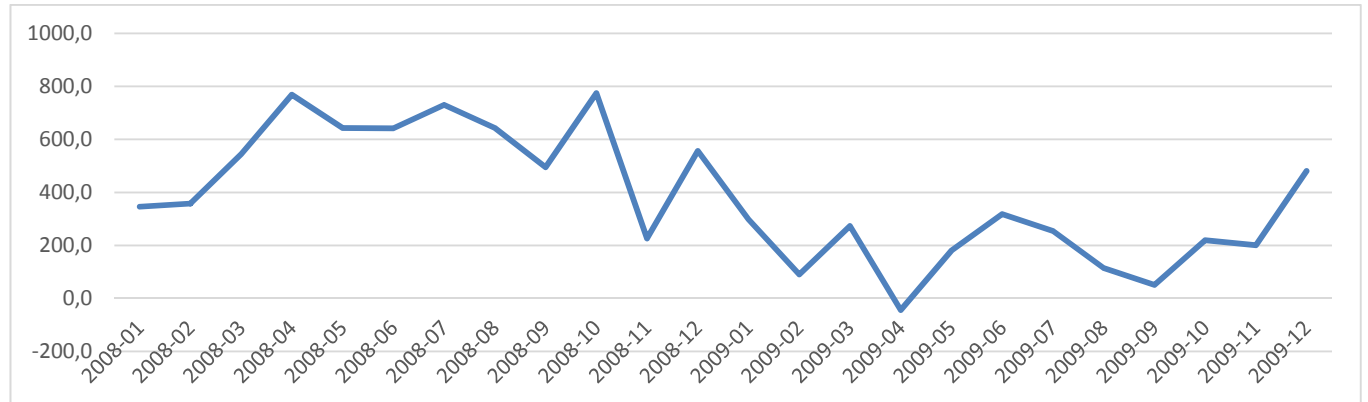

Fig. 8. FDI Bulgaria, 2010-11, monthly data, source Bulgarian National Bank

year filled with many internal political events. Figure 9 demonstrates the clear relationship between them and the dynamics of net FDI transactions:

- on March 13, 2013, after the government resign as a result of protests,an interim government was formed - a sharp decline in FDI;

- The $42^{\text {nd }}$ National Assembly was formed according to the results of the extraordinary parliamentary elections in Bulgaria, held on May 12, 2013. Their first session was on May 21, 2013. The expected stabilization of the internal political situation reflected positively on the growth of FDI;

- on June 14, 2013, Delian Peevsksi was elected as Chairman of the State Agency for National Security (SANS). The started demonstrations, sometimes becoming 


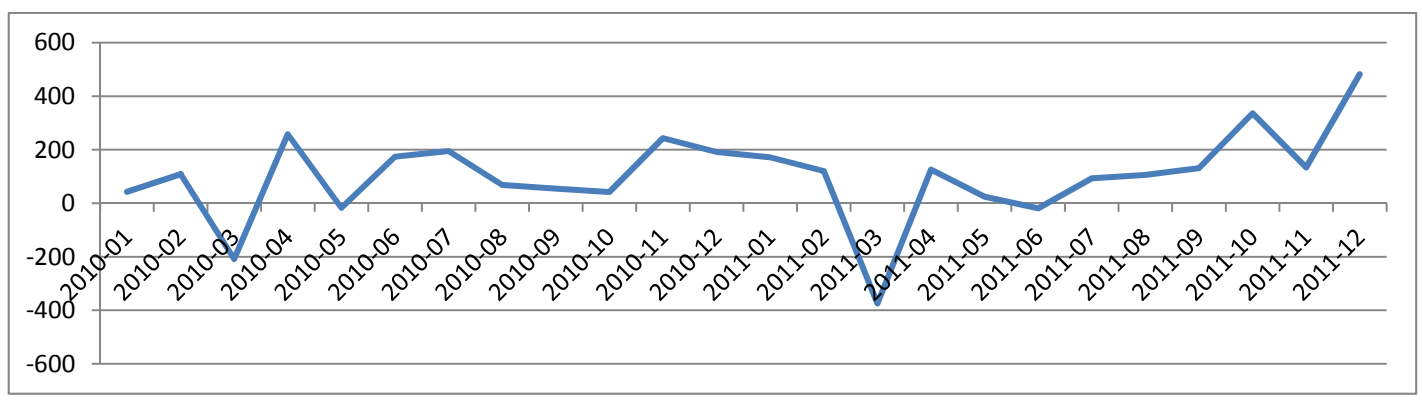

Fig. 9. FDI Bulgaria, 2010-11, monthly data, source Bulgarian National Bank

Table 6. FDI Bulgaria, 2012-2013, monthly data, source Bulgarian National Bank

\begin{tabular}{|c|c|c|c|c|c|c|c|c|c|c|c|c|c|c|c|c|c|c|c|c|c|c|}
\hline \multirow[b]{2}{*}{$\begin{array}{l}\overrightarrow{0} \\
\stackrel{0}{0} \\
0\end{array}$} & \multicolumn{12}{|c|}{$\stackrel{\sim}{\stackrel{\sim}{c}}$} & \multicolumn{10}{|c|}{$\stackrel{m}{\stackrel{n}{c}}$} \\
\hline & $\bar{\sigma}$ & $\widetilde{O}$ & $\hat{\sigma}$ & $\Xi$ & 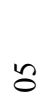 & ๖ & $\hat{0}$ & $\stackrel{\infty}{\circ}$ & o & $\stackrel{ }{ }$ & $=$ & $\simeq$ & $\bar{\sigma}$ & ฮิ & $\hat{0}$ & む & 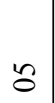 & ๖ & $\hat{0}$ & $\stackrel{\infty}{\circ}$ & oे & $\stackrel{ }{ }$ \\
\hline 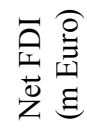 & 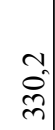 & 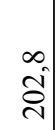 & $\stackrel{\vartheta}{\tilde{I}}$ & $\begin{array}{l}\infty \\
m \\
m\end{array}$ & $\frac{\pi}{7}$ & $\begin{array}{l}? \\
\stackrel{2}{\sim} \\
\stackrel{2}{n}\end{array}$ & $\begin{array}{l}0 \\
\infty \\
\infty\end{array}$ & $\begin{array}{l}0 \\
\curvearrowleft \\
2 \\
m\end{array}$ & $\hat{\imath}$ & $\begin{array}{c}0 \\
\hat{\infty} \\
\infty\end{array}$ & $\begin{array}{l}\infty \\
\underset{1}{ \pm}\end{array}$ & $\begin{array}{l}a \\
\tilde{\sigma} \\
\hat{1}\end{array}$ & $\begin{array}{l}\infty \\
\hat{a}\end{array}$ & ลิ & $\begin{array}{l}0 \\
\infty \\
\infty\end{array}$ & 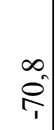 & $\vec{n}$ & $\stackrel{2}{2}$ & 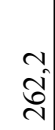 & $\overrightarrow{\mathrm{N}}$ & $\begin{array}{l}N \\
6 \\
n \\
n\end{array}$ & $\frac{\infty}{0}$ \\
\hline
\end{tabular}

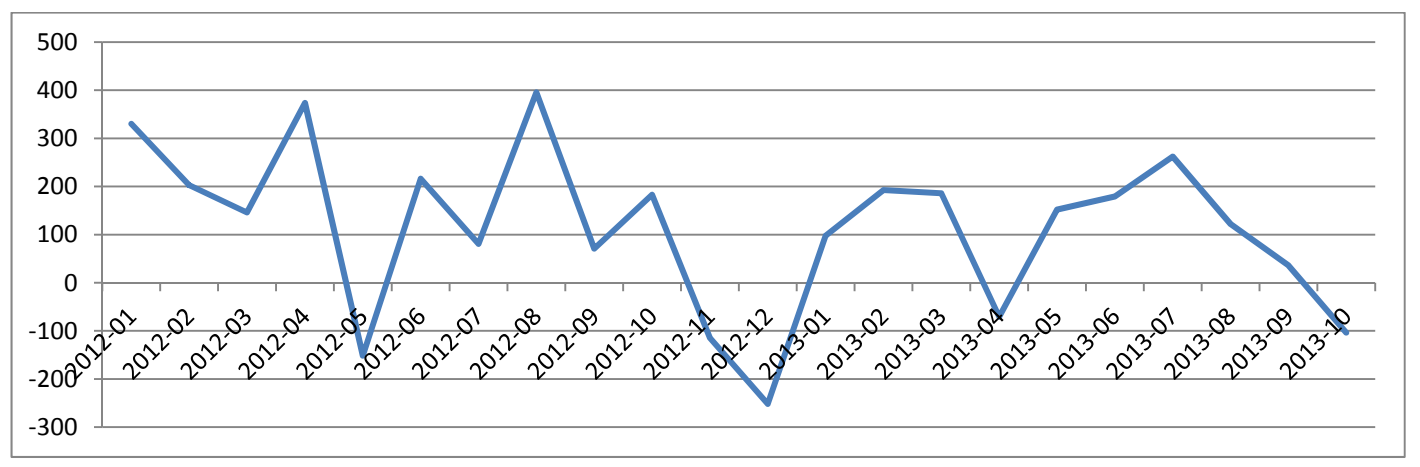

Fig. 10. FDI Bulgaria, 2012-2013, monthly data, source Bulgarian National Bank

riots, were widely disseminated by the media, called into question the legitimacy of the government to the foreign investors. A steady decline in FDI followed, until the end of 2013.

Of the ten major business risks identified and accepted by the World Economic Forum as having the highest importance in 2014, six of them have a direct manifestation in the context of Bulgaria: budget crises; high structural unemployment; severe social stratification; intensification of natural disasters; failure of major financial institutions and mechanisms or profound political and social instability. At the same time, the risk "failure in global governance" is reflected in the crisis "Euromaidan" in Ukraine 


\begin{tabular}{|c|c|c|c|c|c|c|c|c|c|c|c|c|c|c|c|c|}
\hline$\overline{\mathrm{i}}$ & 官 & $\ddot{\sigma}_{0}$ & $\underset{\tau}{\stackrel{1}{\tau}}$ & $\vec{i}$ & $\begin{array}{l}n \\
0 \\
1\end{array}$ & $\begin{array}{l}n \\
i\end{array}$ & $\approx$ & $\overrightarrow{0}$ & $\vec{\Xi}$ & $\begin{array}{l}\vec{i} \\
\stackrel{n}{n}\end{array}$ & $\stackrel{\infty}{\rightarrow}$ & $\begin{array}{l}n \\
\tilde{N}\end{array}$ & $\overline{0}$ & $\hat{\sigma}$ & $\stackrel{+}{\dot{\infty}^{\infty}}$ & $\stackrel{\Xi}{\approx}$ \\
\hline 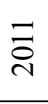 & $\begin{array}{l}\tilde{N} \\
\tilde{\delta} \\
\tilde{n}\end{array}$ & $\begin{array}{l}2 \\
0 \\
\hat{T}_{1}\end{array}$ & $\begin{array}{l}m \\
\Omega^{2}\end{array}$ & $\stackrel{0}{0}$ & $\frac{n}{i}$ & $\begin{array}{l}0 \\
0 \\
0\end{array}$ & $\left|\begin{array}{c}\hat{\infty} \\
-1 \\
T\end{array}\right|$ & $\dot{0}$ & $\stackrel{m}{n}$ & $\hat{m}$ & $\begin{array}{l}0 \\
\text { in }\end{array}$ & $\begin{array}{l}\infty \\
0 \\
1 \\
1\end{array}$ & $\hat{0}$ & $\stackrel{0}{-}$ & $\hat{\text { iे }}$ & $\stackrel{n}{=}$ \\
\hline$\stackrel{\circ}{\stackrel{\sim}{\circ}}$ & $\stackrel{n}{\stackrel{n}{\Xi}}$ & $\begin{array}{l}\hat{\theta} \\
\hat{i}\end{array}$ & $\begin{array}{l}0 \\
= \\
=\end{array}$ & $\begin{array}{l}0 \\
\dot{f}^{\prime}\end{array}$ & $\vec{f}$ & \begin{tabular}{l}
$n$ \\
\multirow{+}{*}{}
\end{tabular} & $\mid \begin{array}{c}\vec{t}_{1} \\
\mathbf{f}_{1}\end{array}$ & $\hat{0}^{\circ}$ & $\begin{array}{l}0 \\
i n\end{array}$ & $\begin{array}{l}1 \\
\infty \\
\omega^{\infty}\end{array}$ & $\overrightarrow{0}$ & $\begin{array}{l}0 \\
0\end{array}$ & 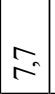 & $0_{0}^{0}$ & $\begin{array}{l}n \\
0 \\
0 \\
0\end{array}$ & $\bar{m}$ \\
\hline \&े & $\mid \begin{array}{l}\hat{\sigma} \\
\hat{\sigma} \\
\text { }\end{array}$ & 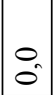 & $\begin{array}{c}0 \\
\infty \\
\infty \\
\sim\end{array}$ & $\hat{0}$ & $\stackrel{\circ}{\circ}$ & $\stackrel{+}{i}$ & $\hat{\vec{i}}$ & $\begin{array}{c}n \\
0\end{array}$ & $\exists$ & $\begin{array}{l}0 \\
\simeq\end{array}$ & $\Rightarrow$ & $\stackrel{\sigma_{f}}{=}$ & $\stackrel{\vec{m}}{\dot{m}}$ & $\stackrel{-}{-}$ & $\tilde{\sigma}^{n}$ & $\stackrel{\sigma}{\Leftrightarrow}$ \\
\hline $\begin{array}{l}\infty \\
\stackrel{\text { }}{0}\end{array}$ & $\begin{array}{l}\infty \\
\stackrel{N}{\hat{\sigma}} \\
\end{array}$ & O & $\begin{array}{l}0 \\
i \\
i\end{array}$ & $\hat{o}$ & $\hat{\sigma}$ & $\because$ & $\vec{m}$ & $\begin{array}{l}0 \\
0 \\
0\end{array}$ & $\begin{array}{l}0 \\
\dot{t} \\
i\end{array}$ & $\begin{array}{l}n \\
m\end{array}$ & 3̂ & $\vec{a}$ & $\overrightarrow{\hat{i}}$ & $\hat{\sigma}$ & $\hat{\sigma}$ & 0 \\
\hline ڤे & \begin{tabular}{l}
$\infty$ \\
$\vec{\sim}$ \\
\multirow{2}{2}{}
\end{tabular} & $\overrightarrow{0}$ & $\begin{array}{l}0 \\
\dot{U} \\
\end{array}$ & $\stackrel{\circ}{-}$ & $\hat{=}$ & $\Rightarrow$ & $\left|\begin{array}{c}\hat{\infty} \\
i\end{array}\right|$ & $\left|\begin{array}{l}\infty \\
0 \\
0\end{array}\right|$ & $\begin{array}{l}\infty \\
i \\
i\end{array}$ & $\vec{m}$ & $O_{0}^{0}$ & $\begin{array}{l}0 \\
\sigma\end{array}$ & $\left|\begin{array}{l}\infty \\
-1\end{array}\right|$ & $\begin{array}{l}\infty \\
0 \\
0\end{array}$ & $\begin{array}{l}n \\
\pm\end{array}$ & $\tilde{0}$ \\
\hline ¿ें & $\mid \begin{array}{l}0 \\
\overrightarrow{\tilde{Z}} \\
\text { (1) }\end{array}$ & 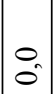 & $\stackrel{m}{m^{2}}$ & $\stackrel{-}{-}$ & $\stackrel{?}{=}$ & $\approx$ & $\left|\begin{array}{l}\hat{\infty} \\
\text { ì }\end{array}\right|$ & $\overrightarrow{0}$ & $\stackrel{2}{\sim}$ & $\hat{n}$ & 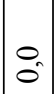 & $\vec{\infty}$ & $\begin{array}{l}n \\
0 \\
0\end{array} \mid$ & $\tilde{\sigma}_{0}$ & $\begin{array}{l}n \\
n\end{array}$ & ? \\
\hline 足 & $\overrightarrow{\hat{n}}$ & 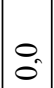 & $\begin{array}{c}\sim \\
\vec{v}\end{array}$ & $\stackrel{0}{0}$ & $\frac{n}{\sim}$ & $\cong$ & $\hat{\hat{\theta}}$ & $\left|\begin{array}{l}0 \\
0 \\
1 \\
1\end{array}\right|$ & $\vec{r}$ & $\begin{array}{l}\infty \\
a\end{array}$ & $\overrightarrow{0}$ & $\begin{array}{l}\sigma_{n} \\
\dot{n}\end{array}$ & $\begin{array}{l}n \\
0 \\
0\end{array}$ & $\begin{array}{l}m \\
0\end{array}$ & $\begin{array}{l}m \\
\infty\end{array}$ & $\cong$ \\
\hline 广 & 官 & $0_{0}$ & $\begin{array}{c}0 \\
\infty^{\circ}\end{array}$ & ? & $\hat{n}$ & $\stackrel{0}{\because}$ & $\stackrel{2}{2}$ & $\begin{array}{l}0 \\
0\end{array}$ & $\begin{array}{l}0 \\
\ddot{n}\end{array}$ & $\begin{array}{l}n \\
\dot{d}\end{array}$ & $\vec{i}^{0}$ & $\begin{array}{l}0 \\
m\end{array}$ & $\vec{f}$ & $\begin{array}{l}n \\
0\end{array}$ & $\vec{\infty}$ & 0 \\
\hline ڤ̊̊̊) & $\begin{array}{l}2 \\
0 \\
0 \\
\infty \\
\infty\end{array}$ & $0_{0}$ & 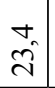 & $\stackrel{\sim}{=}$ & $\hat{\tilde{\lambda}}$ & $\stackrel{?}{\rightarrow}$ & $\begin{array}{l}\infty \\
\infty \\
\infty\end{array} \mid$ & $\left|\begin{array}{l}0 \\
i \\
i\end{array}\right|$ & $\begin{array}{c}m \\
\infty\end{array}$ & $\dot{t}_{0}$ & $\begin{array}{l}0 \\
0\end{array}$ & $\begin{array}{l}n \\
0\end{array}$ & $\vec{m}$ & $\overrightarrow{0}$ & $\hat{n}$ & $\because$ \\
\hline రి & $\begin{array}{l}0 \\
\dot{8} \\
\mathscr{\infty}\end{array}$ & $0_{0}$ & $\begin{array}{l}0 \\
\pm \\
\pm\end{array}$ & $\stackrel{+}{m}$ & $\stackrel{\infty}{\sim}$ & $\hat{\sigma}_{0}$ & {$\left[\begin{array}{l}n \\
0 \\
6\end{array}\right]$} & $\approx$ & $\stackrel{\vec{d}}{\hat{v}}$ & $\approx$ & 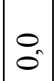 & $\begin{array}{c}0 \\
m\end{array}$ & in & $\tilde{c}$ & $\overrightarrow{0}$ & $\cong$ \\
\hline 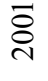 & $\begin{array}{l}+ \\
\tilde{\delta} \\
\tilde{\delta}\end{array}$ & 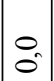 & $\begin{array}{l}n \\
n\end{array}$ & $\stackrel{\infty}{0}$ & $\begin{array}{l}0 \\
\text { ñ }\end{array}$ & $\vec{a}$ & $\left|\begin{array}{c}0 \\
-1\end{array}\right|$ & $\begin{array}{l}\stackrel{f}{f} \\
\text { | }\end{array}$ & हे & $\begin{array}{l}n \\
0\end{array}$ & $\begin{array}{l}0 \\
0\end{array}$ & $\vec{a}$ & $\left|\begin{array}{l}0 \\
i \\
i\end{array}\right|$ & $=$ & in & $\begin{array}{l}0 \\
0\end{array}$ \\
\hline ¿̊ & ?2 & $\begin{array}{l}0 \\
0\end{array}$ & $\stackrel{0}{-}$ & $\stackrel{0}{-}$ & $\begin{array}{l}0 \\
0 \\
0\end{array}$ & $\because$ & $\begin{array}{c}n \\
\tilde{n}^{2}\end{array}$ & $\approx$ & $\stackrel{\infty}{\rightarrow}$ & $\underset{\rightarrow}{\stackrel{\nabla}{i}}$ & $\begin{array}{l}0 \\
0\end{array}$ & $\begin{array}{l}0 \\
i\end{array}$ & $\vec{m}$ & $\begin{array}{l}0 \\
0\end{array}$ & $\hat{\tilde{y}}$ & $\begin{array}{l}0 \\
0\end{array}$ \\
\hline ลे & $\begin{array}{l}0 \\
0 \\
0 \\
0\end{array}$ & ㅇ. & $\stackrel{\partial}{=}$ & $\overrightarrow{0}$ & $\tilde{n}$ & $\begin{array}{l}0 \\
0 \\
i\end{array}$ & $\partial^{2}$ & $\begin{array}{l}0 \\
0 \\
1\end{array}$ & $\stackrel{0}{i}$ & $\begin{array}{l}0 \\
0 \\
1\end{array}$ & $0_{0}$ & $\begin{array}{l}n \\
i \\
i\end{array}$ & 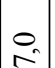 & $\hat{n}$ & $\stackrel{\nabla}{\sigma}$ & 0 \\
\hline হু & $\overline{\hat{b}}$ & $0_{0}^{\circ}$ & $\stackrel{\vec{\nu}}{\vec{v}}$ & $\overrightarrow{0}$ & $\begin{array}{l}n \\
n \\
0 \\
n\end{array}$ & $\begin{array}{c}0 \\
\text { m. }\end{array}$ & $\begin{array}{c}2 \\
6\end{array}$ & 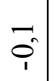 & $\begin{array}{l}\infty \\
i\end{array}$ & $\begin{array}{l}n \\
0\end{array}$ & $\begin{array}{l}0 \\
0\end{array}$ & $\begin{array}{l}n \\
0\end{array}$ & $\begin{array}{l}m \\
0\end{array}$ & $\stackrel{m}{\approx}$ & $\overrightarrow{\vec{N}}$ & O \\
\hline $\begin{array}{l}\overline{0} \\
\stackrel{0}{0} \\
0\end{array}$ & $\underset{\Xi}{\Xi}$ & 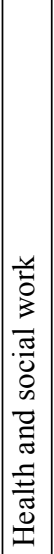 & 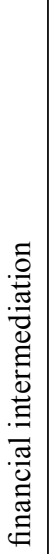 & 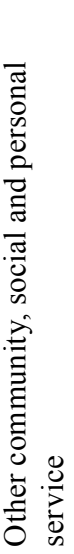 & 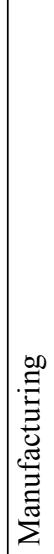 & 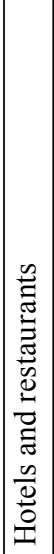 & 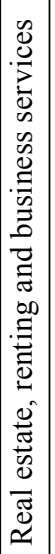 & 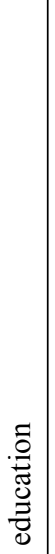 & 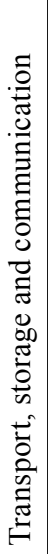 & 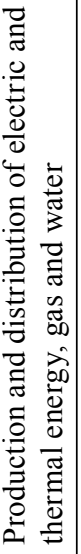 & 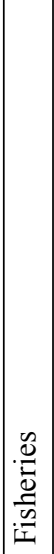 & 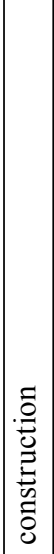 & 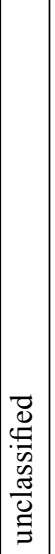 & 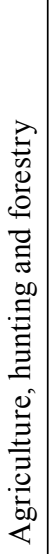 & 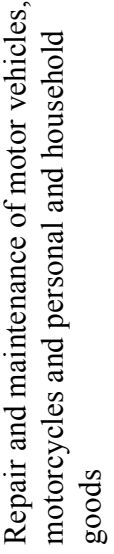 & 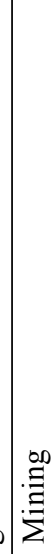 \\
\hline
\end{tabular}


from the end of November 2013. The opposing strategic interests of the United States, the European Union and the Russian Federation lead to failure of the basic principles and mechanisms in inter-state relations formed after the Second World War and polarization of relations between the countries in Eastern Europe. Thus, the domestic crisis has grown to a regional one showing a trend towards extension. Meanwhile, there was polarization concerning the internal political controversies related to FDI originating in the Russian Federation, which approached two billion dollars. ${ }^{7}$ The geopolitical interests of the Russian Federation related to the orthodox cultural space increased. ${ }^{8}$ Logically and contrary to economic logics, the investment process in Bulgaria is politicized, since there is a formed political «elite» associated with so-called EuroAtlantic civilization choice of the country, while there is no established legal mechanism to control the impact of investment activity on national security. (Petrochemical plant «Neftochim» Burgas, owned by the Russian oil company «Lukoil», directly and indirectly provides approximately $25 \%$ of total tax revenues in the Bulgarian budget.) $)^{9}$ This defines the environment of particularly high uncertainty regarding the national risk of the state, and hence comes the negative impact on the dynamics of FDI.

\section{Objects of Direct foreign investments in Bulgaria}

Besides the net value and dynamics of FDI, the economic sectors targeted by the foreign investors are also interesting. The dynamics and the ratio of FDI in the various sectors of the economy is different but the investments dominate in real estate, financial intermediation, manufacturing, production and distribution of electric and thermal energy, gas and water, construction, trade, repair of motor vehicles , motorcycles and personal and household goods.
Investments in agriculture and forestry, health and mining are almost absent. In Figure 11, we see that in the beginning of the period, the investments in manufacturing, trade and financial intermediation are prevailing. Investments in real estate fluctuate from 2 to $13 \%$ out of the total investments. The reason is that this type of investments is not protected by international law in the field of FDI and is influenced very much by the country risk, i.e. the low share of investment in real estate is a direct reflection of internal and regional political and economic uncertainty. After accession to NATO in 2004 and the signing of the Treaty of Accession to the EU in 2005, an overall growth of FDI was observed as well as sustained upward trend in the relative share of FDI in real estate, and in 2008 it exceeded $31 \%$ of total investments.

The data in Figure 12 show that in the beginning of 1998 - 2012, investments in production and distribution of electricity and heat energy practically did not exist (excluding the investment of $2049 \mathrm{~m}$ euro in "Maritza Iztok 1" - Galabovo in 2004, according to the Bulgarian Investment Agency). Since 2008, at the background of an overall decline in FDI, they began to increase, both in absolute value and in relative share of investment in the energy sector. Figures 12 and 13 showed that in 2010, out of all certified investment projects of companies with foreign equity in the industry sector, $96 \%$ were in the renewable energy. The reason for this is the government regulation, stimulating investment in renewable energy. At the same time, this type of foreign investments are protected by international law, which makes them low risk (any change in government regulation affecting investment contracts could be challenged before the arbitration courts).

Within the total FDI volume, the investments in share capital are prevailing (see Fig. 15). The reinvested profit fluctuates around $10 \%$ of the 


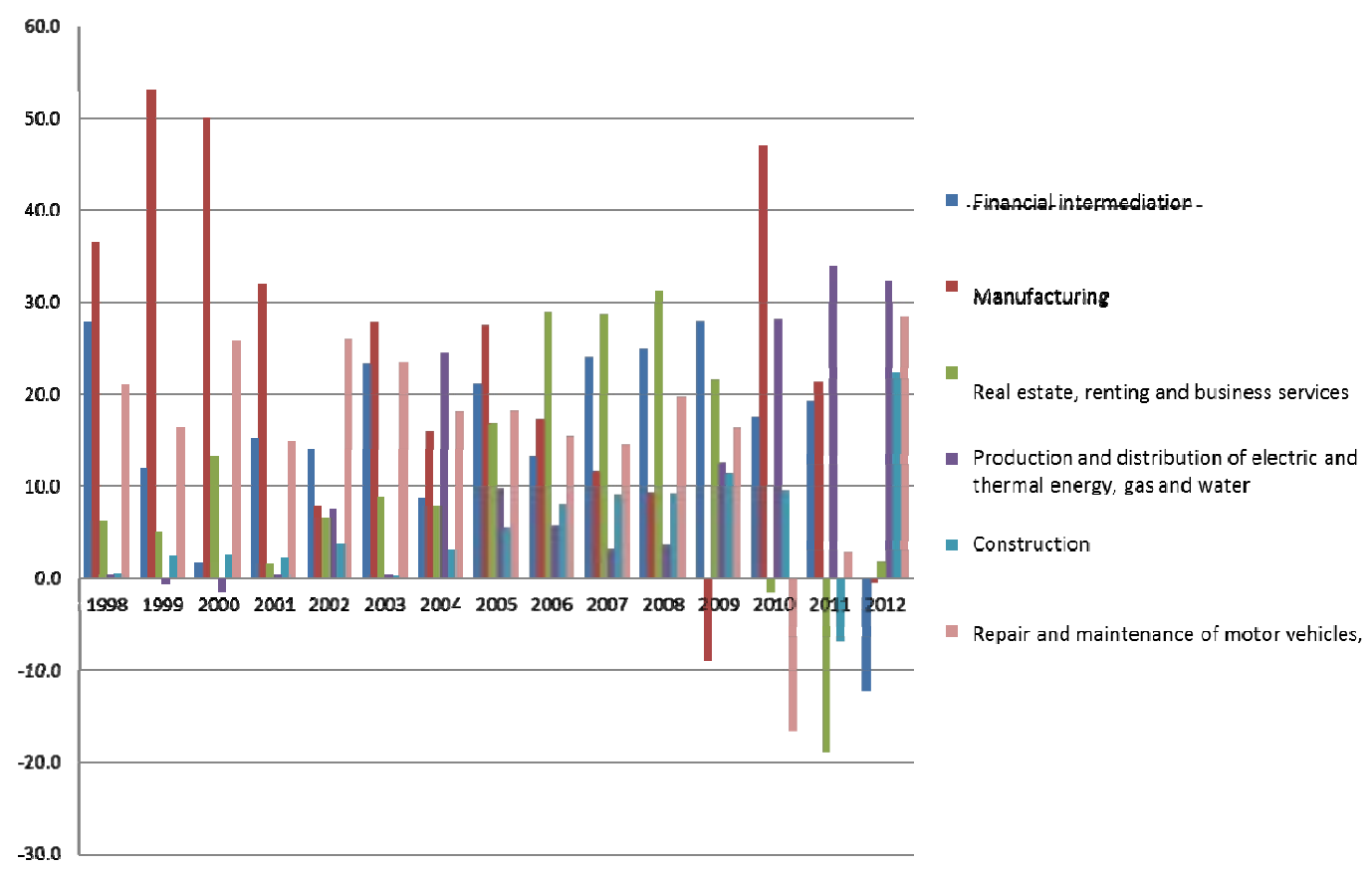

Fig. 11. Percentage of FDI in key economic sectors, 1998 - 2012, source Bulgarian National Bank

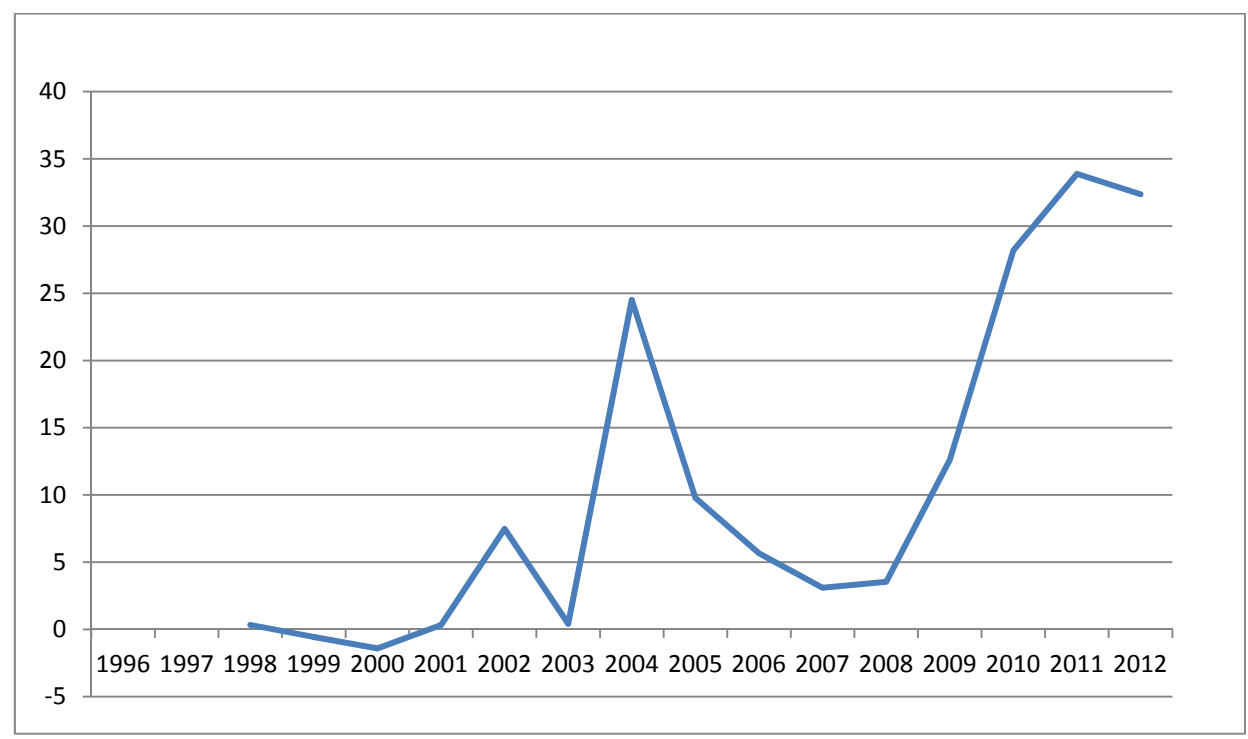

Fig. 12. Percentage ratio of FDI in manufacturing and distribution of electric and heat energy, gaseous fuels and water, 1998 - 2012, source Bulgarian National Bank 


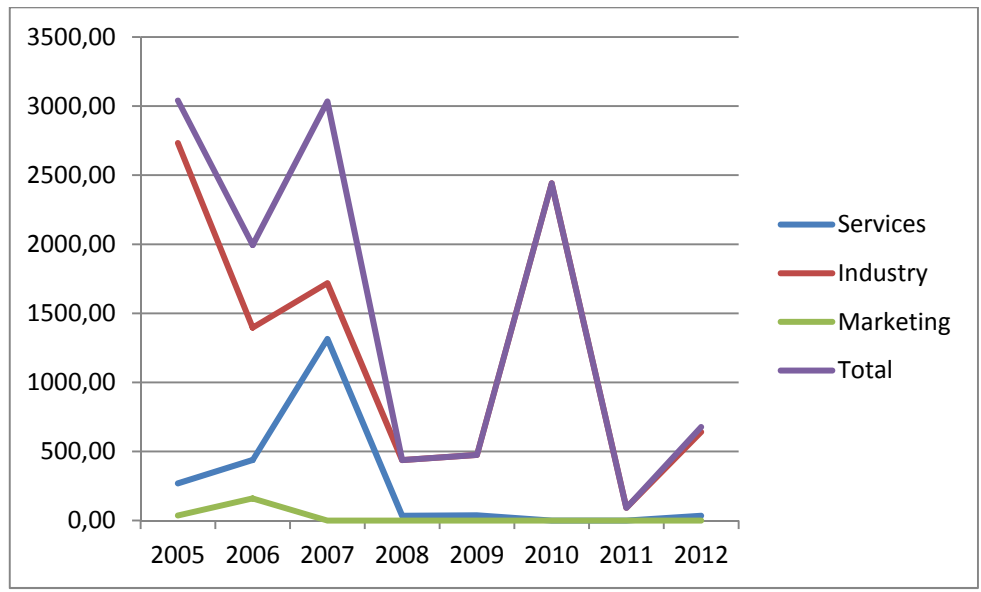

Fig. 13. FDI of companies with foreign interest (Certified projects under the Law on promotion of investments), Bulgarian Agency for Investments

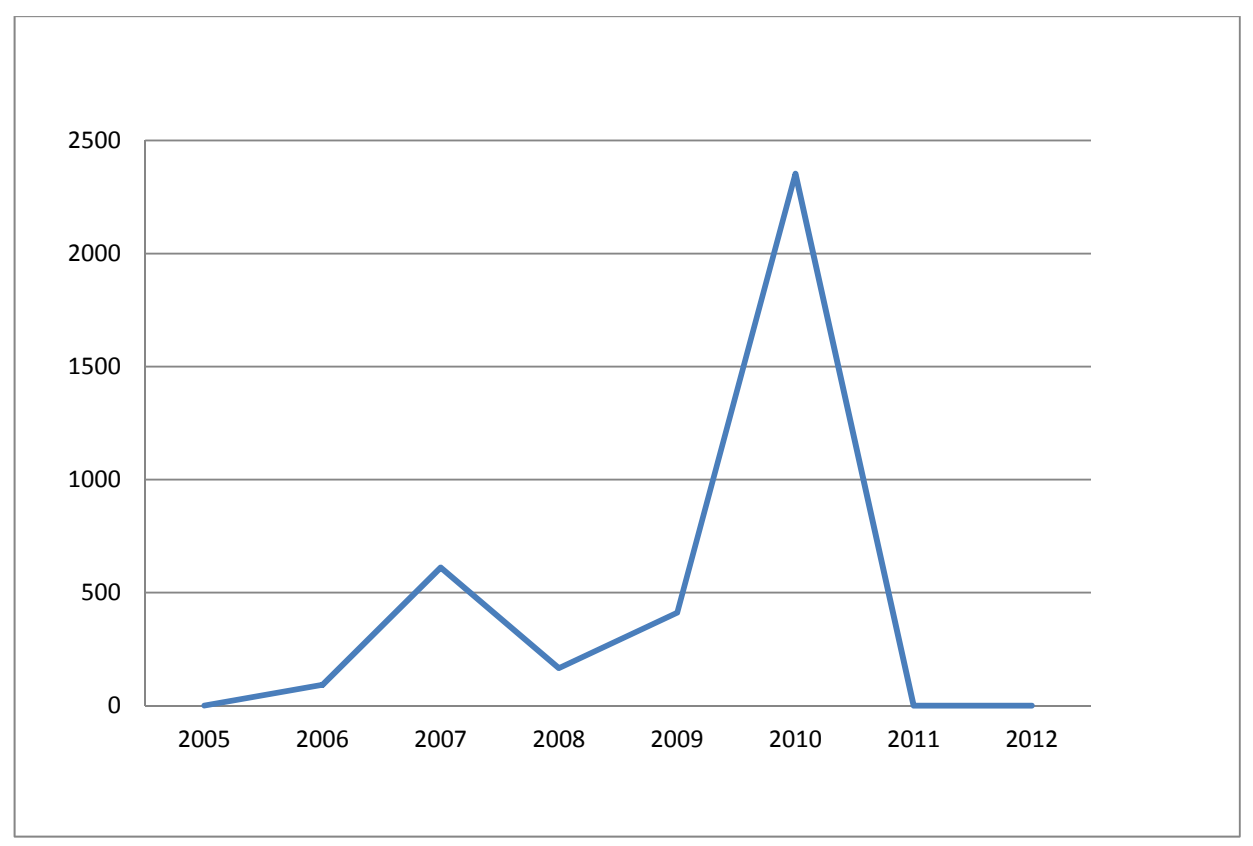

Fig. 14. Value of FDI in renewable energy to entities with foreign interest, the Bulgarian Agency for Investments 
Table 8. FDI by sector, m, annual data, source Bulgarian National Bank

\begin{tabular}{|c|c|c|c|c|c|c|c|c|c|c|c|c|c|c|c|c|c|}
\hline Period & $\stackrel{2}{2}$ & $\hat{a}$ & $\stackrel{\infty}{\varrho}$ & ڤे & 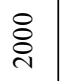 & $\overrightarrow{\stackrel{\sim}{े}}$ & ڤ્ণ & ஸ̊̊̊ & ¿্ণ & 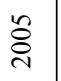 & ঃั & ڤ્ণ & $\stackrel{\text { ڤे }}{\stackrel{\sim}{े}}$ & ڤ્̀े & $\stackrel{ }{\stackrel{i}{*}}$ & $\overline{\vec{\sim}}$ & $\stackrel{\sim}{\stackrel{\sim}{*}}$ \\
\hline FDI, total & $\stackrel{m}{\stackrel{m}{n}}$ & ?ֶ. & $\overline{8}$ & $\begin{array}{l}0 \\
\stackrel{0}{0} \\
\infty\end{array}$ & $\begin{array}{l}\stackrel{m}{0} \\
\stackrel{0}{=}\end{array}$ & $\stackrel{+}{\tilde{Q}}$ & $\begin{array}{l}0 \\
\dot{0} \\
\infty \\
0\end{array}$ & $\begin{array}{l}n \\
0 \\
\infty \\
\infty \\
\infty\end{array}$ & $\begin{array}{l}\hat{c} \\
\hat{n} \\
\text { ते }\end{array}$ & $\begin{array}{l}\vec{i} \\
\vec{n}\end{array}$ & $\begin{array}{l}0 \\
\overrightarrow{\mathbb{J}} \\
0\end{array}$ & $\begin{array}{c}\infty \\
\vec{n} \\
\delta\end{array}$ & $\begin{array}{l}\infty \\
\stackrel{\mathbb{A}}{0} \\
\vdots\end{array}$ & 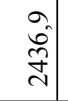 & $\stackrel{\sim}{\stackrel{n}{\leftrightarrows}}$ & 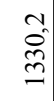 & $\begin{array}{l}n \\
0 \\
\vdots \\
\vdots\end{array}$ \\
\hline $\begin{array}{l}\text { Reinvested } \\
\text { profit }\end{array}$ & & $\stackrel{+}{\circ}$ & $\begin{array}{l}0 \\
\tilde{n}\end{array}$ & f. & $\begin{array}{l}\infty \\
i \\
i\end{array}$ & $\therefore$ & $\begin{array}{l}m \\
\infty \\
\infty \\
\infty\end{array}$ & $\begin{array}{l}0 \\
\text { î } \\
\text {. }\end{array}$ & $\begin{array}{l}\stackrel{+}{*} \\
\dot{f}\end{array}$ & $\begin{array}{c}n \\
0 \\
+ \\
+\end{array}$ & $\begin{array}{l}n \\
\tilde{n} \\
2\end{array}$ & 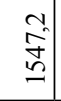 & $\begin{array}{l}n \\
\tilde{\infty} \\
\frac{0}{1}\end{array}$ & $\begin{array}{l}0 \\
\stackrel{0}{0} \\
i\end{array}$ & 告 & $\stackrel{5}{\frac{1}{2}}$ & $\frac{n}{m}$ \\
\hline Equity & $\bar{m}$ & ज़ & 䓹 & $\stackrel{\hat{\sim}}{\text { กे }}$ & $\begin{array}{l}\hat{\infty} \\
\infty \\
\infty\end{array}$ & $\stackrel{\text { ஸे }}{\circ}$ & $\begin{array}{l}0 \\
\overrightarrow{3}\end{array}$ & $\begin{array}{l}\text { r. } \\
\text { है } \\
\text { - }\end{array}$ & $\begin{array}{l}\overrightarrow{0} \\
\infty \\
\infty\end{array}$ & $\begin{array}{l}2 \\
\stackrel{2}{0} \\
=\end{array}$ & 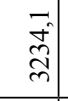 & 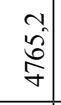 & $\begin{array}{l}\infty \\
0 \\
\sigma \\
\sigma\end{array}$ & $\begin{array}{l}0 \\
+ \\
\infty \\
\infty \\
=\end{array}$ & 胥 & $\begin{array}{l}0 \\
\stackrel{0}{0} \\
=\end{array}$ & $\begin{array}{l}\infty \\
\ddot{0} \\
0\end{array}$ \\
\hline Other capital & कु. & $\hat{\oplus}$ & $\begin{array}{l}\infty \\
\stackrel{\infty}{1}\end{array}$ & $\begin{array}{l}\infty \\
\hat{i} \\
\text { in }\end{array}$ & जी & 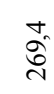 & $\begin{array}{l}\overrightarrow{0} \\
\text { iी }\end{array}$ & ह̂ं & $\begin{array}{l}\hat{\hat{j}} \\
\hat{y}\end{array}$ & $\begin{array}{l}\vec{f} \\
\text { مू }\end{array}$ & $\begin{array}{l}0 \\
\grave{0} \\
\stackrel{0}{0}\end{array}$ & ڤn & 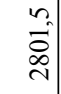 & $\begin{array}{c}0 \\
\hat{\pi} \\
\infty\end{array}$ & $\stackrel{\infty}{\stackrel{\infty}{i}}$ & $\begin{array}{l}m \\
8 \\
q\end{array}$ & 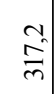 \\
\hline
\end{tabular}

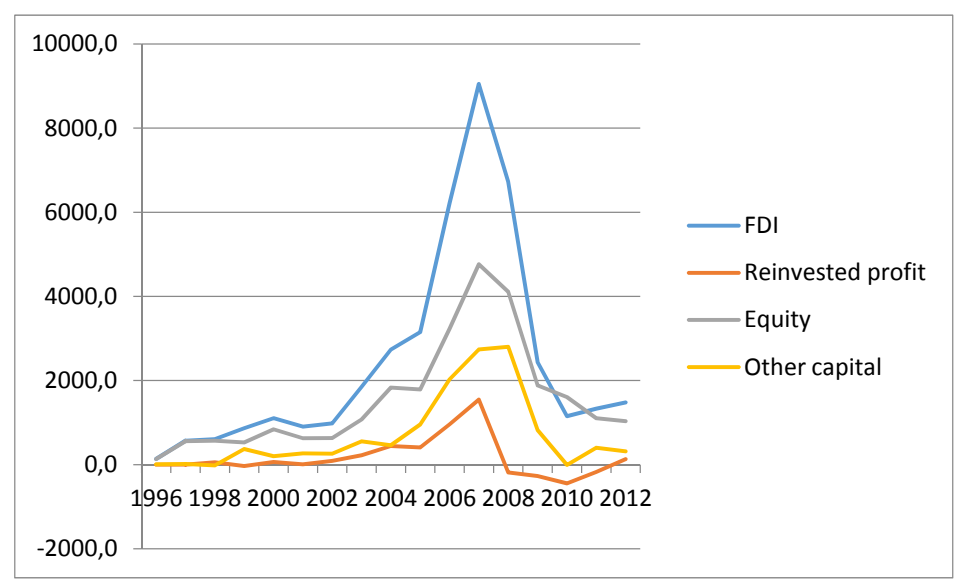

Fig.15. FDI within 1996-2012 by type of investment, source Bulgarian National Bank

total FDI. For the time of the financial crisis, it was negative, and in 2010 it reached $39 \%$ of the total FDI. (See Fig. 16.)

\section{Subjects of Foreign Direct Investment in Bulgaria}

The industrial companies are prevailing among the companies which decided to invest in Bulgaria (see. Figure 13)

As country of origin, the investors are mainly from the Netherlands, Austria and Germany. There is low investment activity of investors from Russia, China and the USA.
Despite the increase in FDI during the period 2005-08 (see Fig. 18), a decrease in the number of newly created jobs is observed. This means that investors switch to capital-intensive industries and investments in long-term tangible assets.

\section{Territorial distribution}

\section{of foreign direct investments in Bulgaria}

In addition to the total number of newly created jobs and the total value of FDI, the interest is focused on their territorial distribution. In Fig. 19, there is a strong imbalance between the South West region 
$\%$

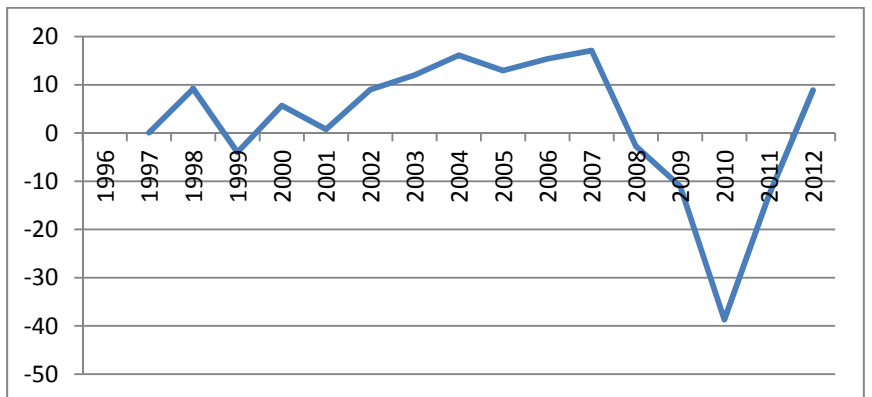

Fig. 16. Reinvested profit, percentage of FDI 1996-2012, source Bulgarian National Bank

Table 9. Distribution of firms by sector, source Goev, V. (2009)

\begin{tabular}{|l|c|c|}
\hline & Number & $\%$ \\
\hline Industry & 48 & 48,5 \\
\hline Commerce & 21 & 21,2 \\
\hline Finance & 6 & 6,1 \\
\hline Tourism & 3 & 3,0 \\
\hline Transport & 8 & 8,1 \\
\hline Communications & 2 & 2,0 \\
\hline Construction & 5 & 5,1 \\
\hline Others & 6 & 6,1 \\
\hline Total & 99 & 100,0 \\
\hline
\end{tabular}

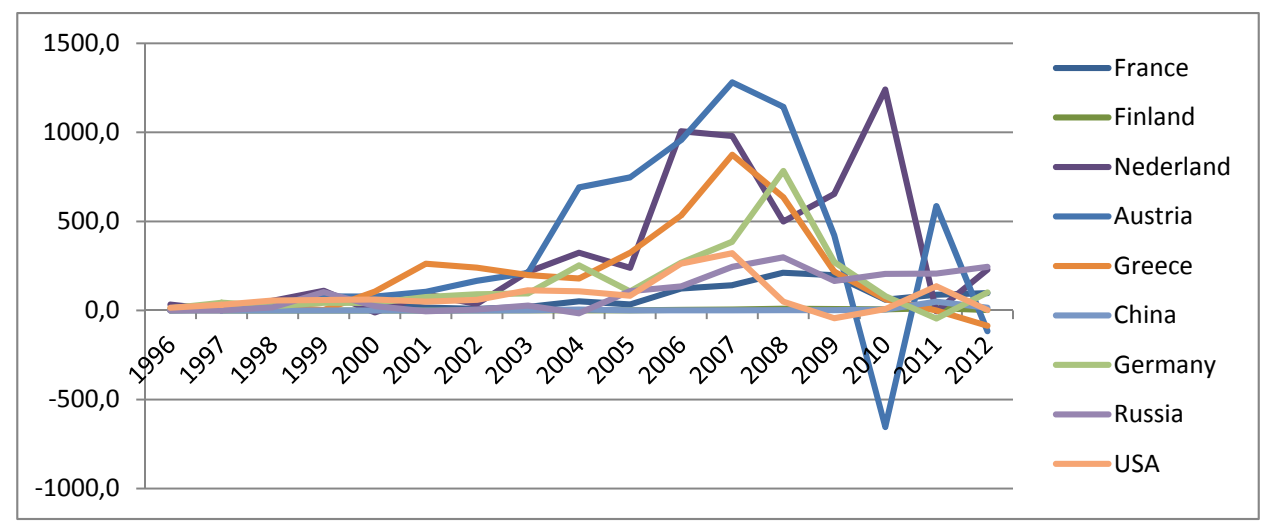

Fig. 17. FDI by country of origin, 1996-2012, source Bulgarian National Bank 


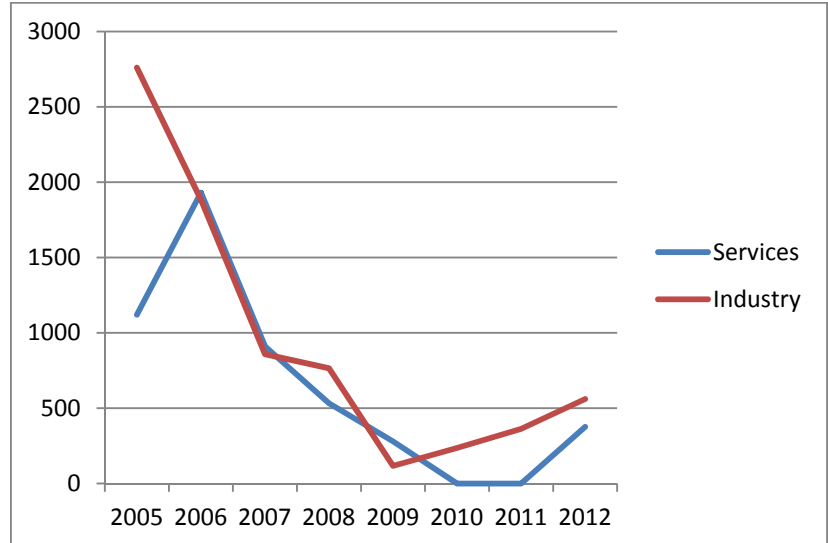

Fig. 18. Number of new jobs as a result of certified investment projects of companies with foreign interest, source Bulgarian Agency for Investments

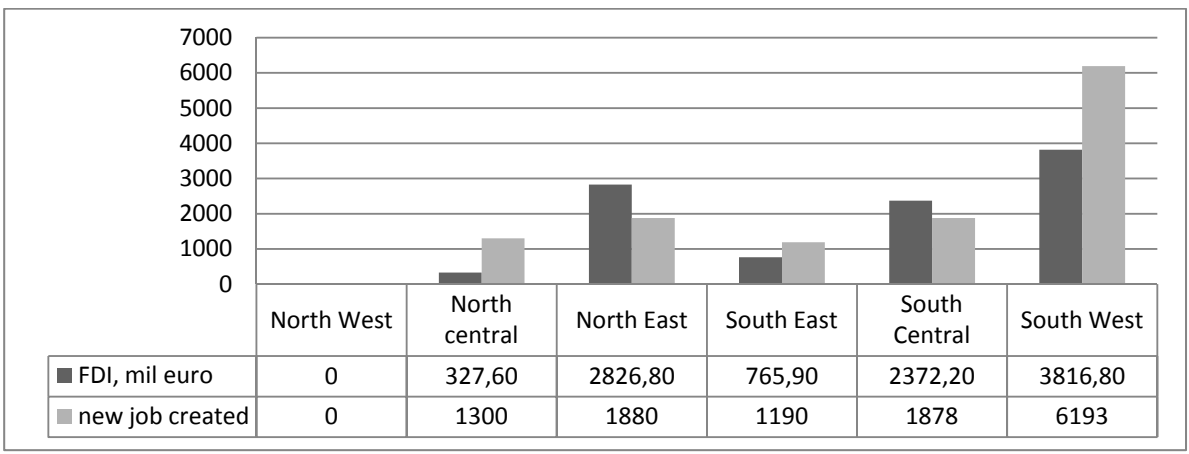

Fig. 19. Territorial distribution of FDI and new jobs created, 2004-12, data by the Bulgarian Agency for Investments

and the rest of the country. The new jobs created exceed three times the other regions. Particularly drastic are the data for the North West Region, where there are no FDI for the period 2004-12, and no jobs were created respectively.

Thus, despite the measures envisaged in the Law on Investment Promotion stimulating FDI in certain municipalities, there is a territorial imbalance leading to a slowdown in the regional development and deepening of the social problems accompanied by increased migration, demographic collapse and further deterioration of the investment potential.

\section{Conclusion}

The historical transition which Bulgaria made from planned to market economy offers a comprehensive information demonstrating and enriching the theoretical aspects of the investment process. The trends in FDI in the period 1999 2013 were examined according to the official data of the Bulgarian National Bank (BNB), UNCTAD and Bulgarian Investment Agency. Analysis of FDI dynamics identified three major periods: the first - from 1999 to 2003, is characterized by the low rates of increase of funds from FDI in the Bulgarian economy; the second - from 2004 to 2007, shows extremely high rates of FDI inflows 
(reach second place in the world index of actual performance in FDI); the third - from 2008 to 2013, noted a sharp decline in the flow of foreign investment. The low level of FDI in the period prior to the entry of the country into NATO and the EU is resulting from the high national risk of the state in all its aspects - political, economic, financial and cultural. The significant growth of FDI in the period 2005-08 was a consequence of the overall condition of the economic cycle, the internal and external political, economic and financial stability. The overall decline in the level of FDI since 2008 is the result of the operation of a global factor such as The global financial crisis and regional factor, the debt and fiscal crisis in Greece (2010) and the crisis "Euromaidan" in Ukraine.

Together with the dependence on the sovereign risk of the country, FDI in Bulgaria are characterized by strong territorial imbalances as well. Major investments are concentrated in the South-Western region, while in the NorthWestern they are practically absent. This causes a delay in regional development and deepening of social problems accompanied by increased migration processes and demographic collapse and further deterioration of the investment potential.

Goev Valentine. 2010 . "Relocation of the West European enterprises in Bulgaria (results of a survey among foreign investors)":, Volume II, - Research Papers - World Economy, Sofia, p.77-114. (http://research.unwe.bg/br19/03.pdf)

2 Sulstarova A., Division on Investment and Enterprise, UNCTAD (http://www.oecd.org/investment/globalforum/44246319. pdf)

3 Kovaleva I. Investment attractiveness as a strategy for the development of regional industry subcomplex APK, Bulletin of the Altai State Agrarian University, No 3 (29), 2007, p.84

4 At the end of 1998, NATO warns President Milosevic to withdraw troops. Then negotiations followed between President Milosevic and US officials for the Balkans Richard Holbrooke. They were unsuccessful and on 24 March 1999 a military strike was launched that lasted 78 days, and then a UN mission was established in Kosovo.

5 On April 18, 2002 in Athens, defense ministers of Bulgaria, Greece, Romania and Turkey make a joint statement on the unified position of the four countries that by the accession of Bulgaria and Romania into NATO will be realized an opportunity to strengthen the Southern flank of the Alliance and strengthening of the Euro-Atlantic security and stability. On November 21, 2002 at the meeting in Prague was decided Bulgaria, together with six other candidate countries to be invited to start negotiations for accession to North Atlantic Alliance.

6 On 19 April $200138^{\text {th }}$ National Assembly was suspended, the $39^{\text {th }}$ National Assembly operates from 5 June 2001 to 17 June 2005, the $40^{\text {th }}$ National Assembly - from 25 June 2005 to 25 June 2009, the $41^{\text {st }}$ National Assembly - from 14 July 2009 to 11 March 2013 and 42 $2^{\text {nd }}$ National Assembly began work on May 21, 2013.

7 http://www.focus-news.net/news/2014/07/07/1940630/ministar-sergey-lavrov-za-10-godini-ruskite-investitsii-v-balgariya-priblizhiha-2-mlrd-dolara-okolo-300-000-rusnatsi-veche-pritezhavat-nedvizhimi-imoti-v-stranata.html

8 Reuters: TsvetanVassilev can buy an Austrian bank <http://www.dnes.bg/business/2014/07/09/roiters-cvetan-vasilevmoje-da-kupi-avstriiska-banka.231728>

9 http://www.focus-news.net/news/2014/07/07/1940630/ministar-sergey-lavrov-za-10-godini-ruskite-investitsii-v-balgariya-priblizhiha-2-mlrd-dolara-okolo-300-000-rusnatsi-veche-pritezhavat-nedvizhimi-imoti-v-stranata.html

\section{References}

Goev Valentine. 2010 . "Relocation of the West European enterprises in Bulgaria (results of a survey among foreign investors)":, Volume II, - Research Papers - World Economy, Sofia, p.77-114. (http://research.unwe.bg/br19/03.pdf).

Kovaleva I. 2007. "Investment attractiveness as a strategy for the development of regional industry sub complex APK", Bulletin of the Altai State Agrarian University, No 3 (29).

Sulstarova A. Division on Investment and Enterprise, UNCTAD

(http://www.oecd.org/investment/globalforum/44246319.pdf)

http://www.focus-news.net/news/2014/07/07/1940630/ministar-sergey-lavrov-za-10-godiniruskite-investitsii-v-balgariya-priblizhiha-2-mlrd-dolara-okolo-300-000-rusnatsi-veche-pritezhavatnedvizhimi-imoti-v-stranata.html 
http://www.focus-news.net/news/2014/07/07/1940630/ministar-sergey-lavrov-za-10-godiniruskite-investitsii-v-balgariya-priblizhiha-2-mlrd-dolara-okolo-300-000-rusnatsi-veche-pritezhavatnedvizhimi-imoti-v-stranata.html

Reuters: "Tsvetan Vassilev can buy an Austrian bank" (http://www.dnes.bg/business/2014/07/09/ roiters-cvetan-vasilev-moje-da-kupi-avstriiska-banka.231728)

\title{
Некоторые особенности инвестиционного процесса и международные инвестиционные проекты в условиях болгарской экономики и политики
}

\author{
Иво Веселинов Йоцов, Сияна Люцканова \\ ВВМУ «Н.Й. Вапиаров» \\ Болгария, 9026, Варна, ул. Васила Друмева, 73
}

\begin{abstract}
В иентре внимания - анализ данных о некоторых иностранных инвестициях (ПИИ) в Болгарии в период 1998-2013 г2. и вопросы, связанные с характеристиками их относительных преимуществ при распределении такой деловой активности иностранных инвесторов в Болгарии. Исторический переход, который Болгария совершила от плановой к рыночной экономике, дает обширный исследовательский материал о влиянии суверенного риска, о развитии инвестиичонного процесса, в частности динамики ПИИ. Проанализировань тенденции в области прямых иностранных инвестиций в период 1999-2013 г2. по официальнылм даннылм Болгарского народного банка (БНБ), ЮНКТАД и Инвестиционного агентства Болгарии. Анализ показал три основных периода: 1) с 1999 по 2003 г., с 2004 по 2007 г. и 3) с 2008 по 2013 г., который характеризуется резким снижением потока иностранных инвестиций. Низкий уровень прямых иностранных инвестиций в период до вступления в ЕС и НАТО является высоким национальным риском во всех его аспектах - политическом, экономическом, финансовом и культурном. Значительный рост ПИИ в период 2005-2008 г2. был связан с общим состоянием экономического ичикла, внутренней и внешней политической, экономической и финансовой стабильностью.
\end{abstract}

Ключевые слова: прямые иностранные инвестиции, экономический рост, экономическая активность регионов.

Научная специальность: 08.00.00 - экономические науки, 23.00.00 - политология. 